\title{
The Association of Virulence Determinants of Uropathogenic Escherichia coli With Antibiotic Resistance
}

\author{
Sara Asadi ${ }^{1}$; Mohammad $\operatorname{Kargar}^{2,}$; Kavous Solhjoo ${ }^{1}$; Akram Najafi ${ }^{3}$; Sadegh Ghorbani- \\ Dalini $^{4}$ \\ ${ }^{1}$ Department of Parasitology, School of Medicine, Jahrom University of Medical Sciences, Jahrom, IR Iran \\ ${ }^{2}$ Department of Microbiology, Jahrom Branch, Islamic Azad University, Jahrom, IR Iran \\ 3 Department of Marine Microbiology, The Persian Gulf Marine Biotechnology Research Center, Bushehr University of Medical Sciences, Bushehr, IR Iran \\ ${ }^{4}$ Department of Microbiology, Jahrom Branch, Young Researcher's Club, Islamic Azad University, Jahrom, IR Iran \\ ${ }^{*}$ Corresponding author: Mohammad Kargar, Department of Microbiology, Jahrom Branch, Islamic Azad University, Jahrom, IR Iran. Tel: +98-917314 9203, Fax: +98-7116476101, E-mail: \\ mkargar@jia.ac.ir
}

Received: January 17, 2013; Revised: April 25, 2013; Accepted: May 5, 2013

\begin{abstract}
Background: The emergence of antimicrobial resistant strains of Escherichia coli has raised considerable interest in understanding the diversity and epidemiology of $E$. coli infections in humans. Virulence factors of E. coli determine the specific infections caused by this microorganism.

Objectives: This study aimed to determine the prevalence of eight E. coli virulence factors and their association with antimicrobial resistance in bacteria isolated from patients with urinary tract infections (UTI).

Patients and Methods: One thousand patients with UTI were enrolled in this cross-sectional study. Antimicrobial susceptibility was examined by disc diffusion method according to CLSI guidelines. After DNA extraction, the materials were probed by PCR for eight virulence factors genes, namely fimH, hly, iucC, ibeA, sfalfoc, neuC, papC, and afa genes.

Results: The frequency of virulence factors papC, afa, sfalfoc, fim H, hly, neuC, ibeA, and iucC were $53.3 \%, 51.7 \%, 53.3 \%, 56.7 \%, 23.3 \%, 31.7 \%, 20 \%$, and 73.3\%, respectively. In addition, there was a high degree resistance to cotrimoxazole and nalidixic acid while a high degree of susceptibility to nitrofurantoin was detected. There was a statistically significant association between $f i m H$ gene and resistance to ciprofloxacin ( $\mathrm{P}=$ $0.006)$, nalidixic acid $(\mathrm{P}=0.025)$, and cotrimoxazole $(\mathrm{P}=0.02)$. Such associations were found between $i$ beA gene and amikacin $(\mathrm{P}=0.02)$ and cotrimoxazole $(\mathrm{P}=0.02)$ as well as between afa gene and gentamycin $(\mathrm{P}=0.05)$.

Conclusions: The results showed that $E$. coli isolated from patients with UTI had eight virulence factors with high frequencies. Moreover, these results alleged a direct connection between virulence factors and antimicrobial resistance in E. coli.
\end{abstract}

Keywords:Escherichia coli; Virulence Factor; Drug Resistance

\section{Background}

The incidence of urinary tract infection (UTI) is estimated to be about 150-250 million cases worldwide. It also accounts for approximately $35 \%$ of all hospital acquired infections $(1,2)$. Escherichia coli is one of the most common agent causing extra intestinal infections. These infections are an important cause of morbidity, mortality, and increased healthcare costs. In addition, they are common leading causes of UTI, pneumonia, meningitis, osteomyelitis, sepsis, and intra-abdominal as well as diverse soft tissue infections.

E. coli strains causing UTI are termed uropathogenic $E$. coli (UPEC). UPEC isolates are a genetically heterogeneous group that possess several virulence factors (VFs) necessary for persistence and colonization of the bacteria in the urinary tract, overcome host defenses, and extra intestinal disease (3-5). These VFs include fimbrial adhesins
(P, type 1, S, and F1C fimbriae), afimbrial adhesin, toxins (hemolysin and cytotoxic necrotizing factor), siderophores (aerobactin system), and capsular polysaccharide (group II capsules) (5-7).

Non-complicated infections constitute the majority of UTIs. Patients recognized with acute non-complicated cystitis are treated as outpatients. The microbiological features of this infection are greatly predictable even in healthy subjects. Therefore, physicians have been informed that empirical antibiotic treatment without culture is convenient in such cases. The empirical therapy has been so widely used that only a few UTIs are routinely cultured (1). Worldwide data shows that there is an increasing resistance to conventional drugs among UTI pathogens. Resistance has emerged even to the newer and more potent antimicrobial agents. Antimicrobial re- 
sistance surveillance is necessary in order to determine the significance of the problem and to guide empirical selection of antimicrobial agents to treat infected patients (2).

During the last few decades, the frequency of antibiotic resistant infections have raised permanently around the world. This increase has been attributed to a combination of the selective pressure of antimicrobial use, microbial characteristics, social and technical changes that accelerate the transmission of resistance factors in microorganisms including misuse and increased use of antibiotics, a higher number of susceptible hosts, and mistakes in infection control programs leading to incremented transmission of resistant microorganisms (8).

However, several investigations have reported the opposite results. Their studies have shown that according to clinical feature, virulence factor, and antibiotic resistant profile, some E. coli strains tend to be less virulent than susceptible isolates. Furthermore, it remains unclear whether such trends are owing to causal relationships between virulence and resistance or result from co-associated factors (3).

\section{Objectives}

This study was conducted to assess the prevalence of VFs and their association with the antibiotics resistance in patients with UTI.

\section{Patients and Methods}

\subsection{Sample Collection}

In this cross-sectional study, 1000 urine samples from patients referred to Peimanieh Hospital Laboratory in Jahrom between 2010 and 2011 were examined. In addition, basic characteristics data such as age, sex, history of urinary infection, history of antibiotic usage, and history of any hospitalization during the past 28 days were recorded. Written informed consent was obtained from the patients or guardian of each child. Ethic Committee of Islamic Azad University approved all steps of this study.

Standard media, including blood agar and MacConkey agar (Merck, Germany) were used for pathogen isolation. Identification of all isolates was done on the basis of Gram staining and routine biochemical tests including fermentation of lactose, citrate utilization, motility of organism, ability to produce indole, reaction on triple sugar iron (TSI) medium, and hemolysis on blood agar. The organisms were stored at $4{ }^{\circ} \mathrm{C}$ on agar slants and at $-20{ }^{\circ} \mathrm{C}$ in glycerol for further investigations. DNA was extracted from confirmed E. coli strains by using a DNA extraction kit (DNP ${ }^{\mathrm{TM}}$ kit, CinaGen Co., Iran) according to the manufacturer's instructions. The quality of extracted DNA was examined by $1 \%$ agarose gel electrophoresis and concentration of extracted DNA was analyzed by A260/A280 ratio in a biophotometer (Eppendorf, Germany).

\subsection{PCR Method to Determine Virulence Factors}

For identification of VFs, $2 \mu \mathrm{L}$ of extracted DNA was amplified with $1.5 \mathrm{mM} \mathrm{MgCl}_{2}, 0.2 \mathrm{mM}$ deoxynucleoside triphosphates (dNTPs) mixture, $0.2 \mathrm{mM}$ of each primer (Table 1), and $1 \mathrm{U}$ of Taq DNA polymerase (CinaGen, Co., Tehran, Iran). The PCR was performed with a Perkin-Elmer Gene Amp 9600 thermal cycler under the following conditions: initial denaturation for $5 \mathrm{~min}$ at $94{ }^{\circ} \mathrm{C}$ followed by 30 cycles of $30 \mathrm{~s}$ at $94^{\circ} \mathrm{C}, 30 \mathrm{~s}$ annealing at the specific melting temperature of each primer (Table 1), $30 \mathrm{~s}$ at 72 ${ }^{\circ} \mathrm{C}$, and a final extension step of $7 \mathrm{~min}$ at $72{ }^{\circ} \mathrm{C}$. The amplified products were visualized after electrophoresis on a 1.5\% agarose gel stained with ethidium bromide (Figure $1)$.

\subsection{Antimicrobial Susceptibility Testing}

The antimicrobial susceptibilities pattern of $E$. coli strains were assessed using the disk diffusion method on Mueller-Hinton agar (Biorad, Marnes-la-Coquette, France) according to the Clinical and Laboratory Standards Institute (9).

Table 1. The Sequence of Primers and Size of Amplified Products (6)

\begin{tabular}{|c|c|c|}
\hline Gene Sequences & Size, bp & $\mathbf{T m},{ }^{\circ} \mathrm{C}$ \\
\hline fimH & 508 & 55 \\
\hline \multicolumn{3}{|l|}{ F: TGCAGAACGGATCCGTGG } \\
\hline \multicolumn{3}{|l|}{ R: GCAGTCACCTGCCCTCCGGTA } \\
\hline iucC & 269 & 55 \\
\hline \multicolumn{3}{|l|}{ F: AAACCTGGCTTACGCAACTGT } \\
\hline \multicolumn{3}{|l|}{ R:ACCCGTCTGCAAATCATGGAT } \\
\hline papC & 328 & 65 \\
\hline \multicolumn{3}{|l|}{ F: GACGGCACTGCTGCAGGGTGTGGCG } \\
\hline \multicolumn{3}{|l|}{ R: ATATCCTTTCTGCAGGGATGCAATA } \\
\hline sfalfoc & 410 & 65 \\
\hline \multicolumn{3}{|l|}{ F: CGGAGGAGTAATTACAAACCTGGCA } \\
\hline \multicolumn{3}{|l|}{ R: GAGAACTGCCCGGGTGCATACTCT } \\
\hline ibeA & 171 & 60 \\
\hline \multicolumn{3}{|l|}{ F: TTACCGCCGTTGATGTTATCA } \\
\hline \multicolumn{3}{|l|}{ R: CATTAGCTCTCGGTTCACGCT } \\
\hline neuC & 675 & 61 \\
\hline \multicolumn{3}{|l|}{ F: AGGTGAAAAGCCTGGTAGTGTG } \\
\hline \multicolumn{3}{|l|}{ R: GGTGGTACATTCCGGAGTGTC } \\
\hline hly & 1177 & 63 \\
\hline \multicolumn{3}{|l|}{ F: AACAAGGATAAGCACTGTTCTGGCT } \\
\hline \multicolumn{3}{|l|}{ R: ACCATATAAGCGGTCATTCCCGTCA } \\
\hline$a f a$ & 750 & 65 \\
\hline \multicolumn{3}{|l|}{ F: GCTGGGCATCAAACTGATAACTCTC } \\
\hline R: CATCAAGCTGTTTGTTCGTCCGCCG & & \\
\hline
\end{tabular}


Figure 1. Gel Electrophoresis of Virulence Genes of Uropathogenic E. coli.

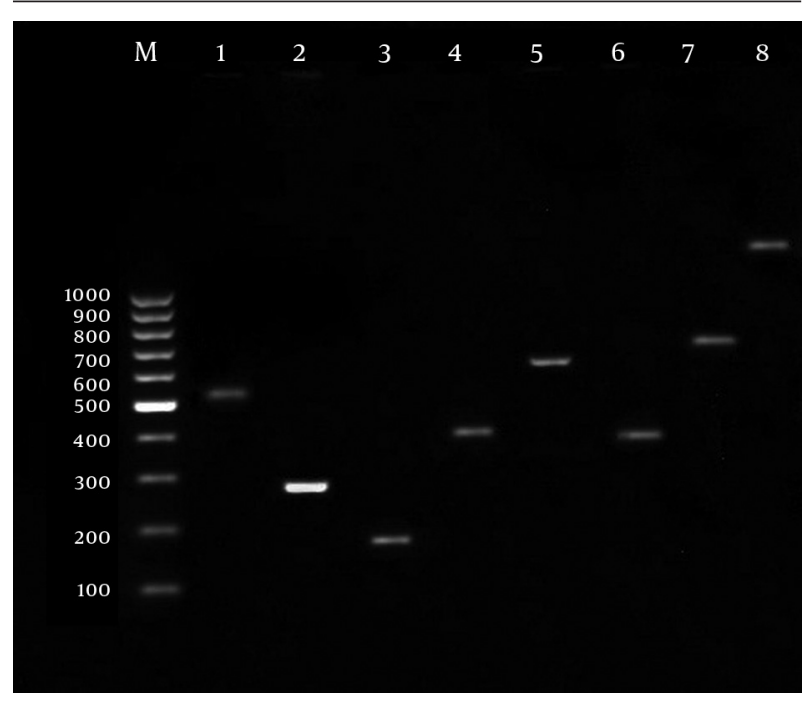

M) Size Marker (100 bp);1) fimH (508 bp); 2) iucC (269 bp); 3) ibeA (171 bp); 4) sfa/foc (410 bp); 5) neuC (675 bp); 6) papC (328 bp); 7) afa (750 bp); 8) hly (1117 bp);

In the present study, the eight used antibiotics were as follows: cotrimoxazole $(1.25 \mu \mathrm{g}$ trimethoprim and $23.75 \mu \mathrm{g}$ sulfamethoxazole), nalidixic acid $(30 \mu \mathrm{g})$, ciprofloxacin (5 $\mu \mathrm{g})$, cefixime $(5 \mu \mathrm{g})$, gentamicin $(10 \mu \mathrm{g})$, cephalexin (30 $\mu \mathrm{g})$, amikacin $(30 \mu \mathrm{g})$, and nitrofurantoin $(300 \mu \mathrm{g})$ (Mast Diagnostics, Merseyside, UK). E. coli strain ATCC 25922 was used as quality control.

\subsection{Statistical Analysis}

Data were statistically analyzed using SPSS v.15(SPSS Inc., Chicago, IL, USA). Descriptive analyses were done to the evaluation of parametric and non-parametric variables. In addition, caramer, fi, and logistic regression were performed to assess of variables correlation. P value $<0.05$ was considered as statistically significant.

\section{Results}

In total, 116 out of 1000 samples showed bacterial growth in culture media including 60 (51.7\%) E. coli, 43 (37.1\%) Klebsiella, 8 (6.9\%) Pseudomonas, and 5 (4.3\%) Proteus. Among 60 UPEC isolates, 47 isolates were from females and 13 were from males. The mean age of the children studied was $33.8 \pm 2.07$ years old; the oldest patient had 90 years of age and the youngest one was one year old. The frequency of each virulence factor was as follows: 19 (31.7\%) neuC (K1capsular antigen), 31 (51.7\%) afa (afimbriae adhesion), 32 (53.3\%) sfalfoc (type S fimbriae), 34 (56.7\%) fimH (type I fimbriae), 32 (53.3\%) papC (type P pilli), 14 (23.3\%) hly (alpha-Hemolysin), 12 (20\%) ibeA (invasive protein A), and 44 (73.3\%) iucC (aerobactin system).

The rate of resistance to cotrimoxazole, nalidixic acid, ciprofloxacin, cefixime, gentamycin, cephalexin, amikacin, and nitrofurantoin antibiotics were 45\%, 41.7\%, 21.7\%, $20 \%, 11.7 \%, 16.7 \%, 13.3 \%$, and $3.3 \%$, respectively (Table 2 ). Statistical analysis revealed the existence of the following associations: between fim $\mathrm{H}$ gene and resistance to ciprofloxacin $(\mathrm{P}=0.006)$, nalidixic acid $(\mathrm{P}=0.025)$, and cotrimoxazole $(\mathrm{P}=0.02)$; between ibeA gene and amikacin $(\mathrm{P}=0.02)$ and cotrimoxazole $(\mathrm{P}=0.02)$; afa gene and gentamycin $(\mathrm{P}=0.05)$ (Table 3$)$. Moreover, there was an association between the hly gene and a history of antibiotic consumption $(\mathrm{P}=0.04)$.

\section{Discussion}

E. coli is the leading causative agent of UTI and one of the most important bacterial infections. In most cases, uropathogenic clones originate from the fecal flora, and the pathogenic potential of $E$. coli isolates is thought to be dependent on the presence of various VFs (5). This study showed that there was a difference in rate of VFs in 60 examined UPEC.

Tiba et al. (5) conducted a study on the genetics of VFs of pathogenic E. coli from patients with cystitis. The highest frequency rates of VFs were consecutively attributed to $\mathrm{fimH}(97.5 \%), \operatorname{papC}(32.7 \%)$, afa $(27.8 \%)$, iucC (25.9\%), hly (25.3\%), and afa (6.2\%). Moreover, the incidence of virulence genes in E. coli strains isolated from Romanian adult with UTI was fimH (86\%), sfalfoc (23\%), papC (36\%), and $a f a(14 \%)$ (7). Andreu et al. (10) identified the presence of fimbriae type I ( $i m H$ ) in E. coli strains isolated from patients with pyelonephritis, cystitis, and recurrent UTI as $97 \%, 97 \%$, and $90 \%$, respectively. In addition, the frequency rate of papC was $73 \%, 0 \%$, and $20 \%$, respectively. In another study, Yasuoka reported that the most frequent E. coli VFs in stool, urine, and blood samples in Japan were ibeA (44\%), papC (45\%), and hlyA (22\%) (11). In a further study by Santo, which was performed on VFs of pathogenic E. coli strains, the prevalence of VFs were reported as sfa $19 \%$, aerobactin $76 \%$, papC $11 \%$, afa $32 \%$, and hemolysin $96 \%$ (12). Blanco reported that the frequency rates of $s f a, p a p C$, and afa VFs in pathogenic strains of E. coli isolated from patients with UTI were 53\%, 54\%, and $2 \%$, respectively (13).

\begin{tabular}{lccc}
\hline \multicolumn{4}{l}{ Table 2. Antibiotic Susceptibility Pattern of Uropathogenic E. coli ${ }^{\text {a }}$} \\
\hline Antibiotics & Sensitive & Resistant & Intermediate \\
\hline Ciprofloxacin & $44(73.3)$ & $13(21.7)$ & $3(5)$ \\
\hline Nalidixic acid & $34(56.7)$ & $25(41.7)$ & $1(1.7)$ \\
\hline Amikacin & $48(80)$ & $8(13.3)$ & $4(6.7)$ \\
Cotrimoxazole & $31(51.7)$ & $27(45)$ & $2(3.3)$ \\
Nitrofurantoin & $58(96.7)$ & $2(3.3)$ & 0 \\
Cefixime & $46(76.7)$ & $12(20)$ & $2(3.3)$ \\
Gentamycin & $48(80)$ & $11(11.7)$ & $5(8.3)$ \\
Cephalexin & $45(75)$ & $10(16.7)$ & $5(8.3)$ \\
\hline
\end{tabular}

${ }^{\mathrm{a}}$ Data are presented in No. (\%). 
Asadi S et al.

\begin{tabular}{|c|c|c|c|c|c|c|c|c|}
\hline Antibiotic & $\mathrm{CN}$ & GM & CFM & FM & SXT & AN & NA & $\mathbf{C P}$ \\
\hline \multicolumn{9}{|c|}{ neuC $(n=19)$} \\
\hline Positive, $\%$ & $2(10.5)$ & $2(10.5)$ & $2(10.5)$ & $0(0)$ & $9(47.4)$ & $2(10.5)$ & $9(47.4)$ & $2(10.5)$ \\
\hline Pvalue & 0.38 & 0.85 & 0.91 & 0.32 & 0.8 & 0.66 & 0.54 & 0.15 \\
\hline \multicolumn{9}{|l|}{$A f a(n=31)$} \\
\hline Positive, \% & $5(16.1)$ & $6(19.4)^{c}$ & $8(25.8)$ & $2(6.5)$ & $14(45.2)$ & $4(12.9)$ & $13(41.9)$ & $6(19.4)$ \\
\hline Pvalue & 0.91 & 0.05 & 0.24 & 0.16 & 0.97 & 0.91 & 0.96 & 0.65 \\
\hline \multicolumn{9}{|c|}{ Sfa/foc $(\mathrm{n}=32)$} \\
\hline Positive, \% & $6(18.8)$ & $4(12.5)$ & $6(18.8)$ & 0 & $13(40.6)$ & $2(6.3)$ & $13(40.6)$ & $6(18.8)$ \\
\hline Pvalue & 0.64 & 0.83 & 0.79 & 0.12 & 0.46 & 0.08 & 0.86 & 0.55 \\
\hline \multicolumn{9}{|c|}{$f i m H(n=34)$} \\
\hline Positive, \% & $4(11.8)$ & $4(11.8)$ & $4(11.8)$ & $1(2.9)$ & $11(32.4)^{C}$ & $6(17.6)$ & $12(35.3)^{C}$ & $3(8.8)^{\mathrm{C}}$ \\
\hline Pvalue & 0.24 & 0.97 & 0.06 & 0.84 & 0.02 & 0.26 & 0.025 & 0.006 \\
\hline \multicolumn{9}{|c|}{$\operatorname{papC}(\mathrm{n}=32)$} \\
\hline Positive, \% & $6(18.8)$ & $3(9.4)$ & $8(25)$ & $1(3.1)$ & $16(50)$ & $3(9.4)$ & $14(43.8)$ & $8(25)$ \\
\hline Pvalue & 0.64 & 0.55 & 0.3 & 0.92 & 0.4 & 0.33 & 0.72 & 0.5 \\
\hline \multicolumn{9}{|l|}{$h l y(n=14)$} \\
\hline Positive, \% & $1(7.1)$ & $2(14.3)$ & $2(14.3)$ & 0 & $5(6.3)$ & $2(14.3)$ & $3(21.4)$ & $1(7.1)$ \\
\hline Pvalue & 0.27 & 0.72 & 0.54 & 0.42 & 0.42 & 0.9 & 0.07 & 0.13 \\
\hline \multicolumn{9}{|l|}{$\operatorname{ibeA}(\mathrm{n}=12)$} \\
\hline Positive, \% & $3(25)$ & $2(16.7)$ & $3(25)$ & 0 & $9(0.75)^{C}$ & $4(33)^{\mathrm{C}}$ & $6(50)$ & $3(25)$ \\
\hline Pvalue & 0.38 & 0.54 & 0.62 & 0.47 & 0.02 & 0.02 & 0.51 & 0.75 \\
\hline \multicolumn{9}{|c|}{$\operatorname{iucC}(n=44)$} \\
\hline Positive, \% & $8(18.2)$ & $6(13.6)$ & $10(22.7)$ & $1(2.3)$ & $19(43.2)$ & $5(11.4)$ & $20(45.5)$ & $10(22.7)$ \\
\hline P value & 0.6 & 0.43 & 0.38 & 0.44 & 0.63 & 0.45 & 0.32 & 0.74 \\
\hline
\end{tabular}

a Abbreviations: AN, Amikacin; CFM, Cefixime; CN, Cephalexin; CP, Ciprofloxacin; FM, Nitrofurantoin; GM, Gentamycin; NA, Nalidixic acid; SXT: Sulfamethoxazole with Trimethoprim (cotrimoxazole).

$\mathrm{b}$ Data are presented in No.(\%).

${ }^{\mathrm{c}}$ Statistically significant

In another study applied by Farshad et al. (14), the prevalence rates of sfa, papC, and hly VFs in children with UTI were $13.5 \%, 22.9 \%$, and $14.6 \%$, respectively. Benton examined E. coli VFs of patients with spinal injuries. The prevalence rates of VFs were as follows: antigen $\mathrm{K}(28 \%)$, aerobactin (39\%), fimbraie P (17\%), and hemolysin (27\%) (15).

The differences observed in the prevalence of $E$. coli VFs in this study and reports from some regions $(7,11,12,14)$ could be explained by the sample type, source, size, type of VFs, and geographic location. Horcajada showed that nalidixic acid resistance was significantly associated with a decrease in prevalence of three VFs, namely sfa, hly, and cnf-1 (16). In another study, Arisoy et al. (17) showed an association between susceptibility to antibiotics and VFs of the E. coli isolates causing pyelonephritis. They reported that virulence genes pap, sfa, afaI, hly, and aer were increased in sensitive strains.

Zhao examined the prevalence of VFs and antibiotic resistance in UPEC. The $f e o B$ and $f i m H$ genes had the highest prevalence. in addition, among the 15 tested antibiotics for resistance, nalidixic acid, mezlocillin, and tetracycline had the most resistance (18). In this study, stronger associations were found between $\mathrm{fimH}$ gene and resistance to ciprofloxacin, nalidixic acid, and cotrimoxazole, between ibeA gene and amikacin and cotrimoxazole, and between afa gene and gentamycin. These findings were similar to those reported in other studies (16-18).

Antimicrobial susceptibility patterns varied in isolates from different categories of patients. it needs to be considered when developing guidelines for treatment of UTI and interpreting data from other published studies, which showed high prevalence of antimicrobial resistance among UPEC. From our data, older drugs like nitrofurantoin appeared to be useful and could be considered as a choice for treating uncomplicated lower UTI.

To sum up, E. coli causing UTI in different patient populations differ in their pathogenic potential and susceptibility to antimicrobial agents. It has to be considered 
when developing guidelines for management of UTI. Periodic reviews and formulation of antibiotic consumption policy are required to control the transmission and acquisition of antibiotic resistance. Further studies for better understanding of the interaction between different VFs at a molecular level are necessary as most UPEC isolates simultaneously express several VFs.

\section{Acknowledgements}

AcknowledgementsThe authors expressed their thanks and appreciation to the Islamic Azad University, Jahrom branch and the staffs of Peimanieh Hospital laboratory for their executive participation in this project.

\section{Authors' Contribution}

Study concept and design: Mohammad Kargar and Kavous Solhjoo; analysis and interpretation of data: Mohammad Kargar, Sara Asadi, Kavous Solhjoo, and Sadegh Ghorbani-Dalini; drafting of the manuscript: Sara Asadi and Akram Najafi; collection, assembly, possession of raw data: Mohammad Kargar, Sara Asadi, and Kavous Solhjoo; final approval of the study: Mohammad Kargar, Sara Asadi, and Akram Najafi; statistical analysis: Sadegh Ghorbani-Dalini.

\section{Financial Disclosure}

None declared.

\section{Funding/Support}

This work had no fund.

\section{References}

1. Santo E, Salvador MM, Marin JM. Multidrug-resistant urinary tract isolates of escherichia coli from Ribeirao Preto, Sao Paulo, Brazil. Braz JInfect Dis. 2007;11(6):575-8.

2. Hassan SA, Jamal SA, Kamal M. Occurrence of multidrug resistant and ESBL producing E. coli causing urinary tract infections. $J$ of Basic and Applied Sci. 2011;7(1):39-43.

3. Johnson JR, Kuskowski MA, Gajewski A, Sahm DF, Karlowsky JA. Virulence characteristics and phylogenetic background of multidrug-resistant and antimicrobial-susceptible clinical isolates of Escherichia coli from across the United States, 2000-2001. J Infect Dis. 2004;190(10):1739-44.
4. Johnson JR, Kuskowski MA, Gajewski A, Soto S, Horcajada JP, Jimenez de Anta MT, et al. Extended virulence genotypes and phylogenetic background of Escherichia coli isolates from patients with cystitis, pyelonephritis, or prostatitis. J Infect Dis. 2005;191(1):46-50.

5. Tiba MR, Yano T, Leite Dda S. Genotypic characterization of virulence factors in Escherichia coli strains from patients with cystitis. Rev Inst Med Trop Sao Paulo. 2008;50(5):255-60.

6. Ghada D, Suzuki Y, Yamamoto T, Suzumori N, Takashi S, Wakimoto Y, et al. An Approach for Detection of Escherichia coli by the PCR technique.J Nagoya Med. 2007;49:17-25.

7. Usein CR, Damian M, Tatu-Chitoiu D, Capusa C, Fagaras R, Tudorache D, et al. Prevalence of virulence genes in Escherichia coli strains isolated from Romanian adult urinary tract infection cases. J Cell Mol Med. 2001;5(3):303-10.

8. Dzidic S, Bedekovic V. Horizontal gene transfer-emerging multidrug resistance in hospital bacteria. Acta Pharmacol Sin. 2003;24(6):519-26.

9. Cockerill FR. Performance standards for antimicrobial susceptibility testing: twenty-first informational supplement.Wayne, PA: Clinical and Laboratory Standards Institute (CLSI); 2011.

10. Andreu A, Xercavins M, Fernandez F. [Type 1 fimbriae, P fimbriae and $\mathrm{X}$ adhesins in Escherichia strains causing pyelonephritis, cystitis and recurrent urinary infections]. Med Clin (Barc). 1989;92(11):409-12.

11. Obata-Yasuoka M, Ba-Thein W, Tsukamoto T, Yoshikawa H, Hayashi $H$. Vaginal Escherichia coli share common virulence factor profiles, serotypes and phylogeny with other extraintestinal E. coli. Microbiology. 2002;148(Pt 9):2745-52.

12. Santo E, Macedo C, Marin JM. Virulence factors of uropathogenic Escherichia coli from a university hospital in Ribeirao Preto, Sao Paulo, Brazil. Rev Inst Med Trop Sao Paulo. 2006;48(4):185-8.

13. Blanco M, Blanco JE, Alonso MP, Mora A, Balsalobre C, Munoa F, et al. Detection of pap, sfa and afa adhesin-encoding operons in uropathogenic Escherichia coli strains: relationship with expression of adhesins and production of toxins. Res Microbiol. 1997;148(9):745-55.

14. Farshad S, Emamghorashi F. The prevalence of virulence genes of E. coli strains isolated from children with urinary tract infection. Saudi J Kidney Dis Transpl. 2009;20(4):613-7.

15. Benton J, Chawla J, Parry S, Stickler D. Virulence factors in Escherichia coli from urinary tract infections in patients with spinal injuries. J Hosp Infect. 1992;22(2):117-27.

16. Horcajada JP, Soto S, Gajewski A, Smithson A, Jimenez de Anta MT, Mensa J, et al. Quinolone-resistant uropathogenic Escherichia coli strains from phylogenetic group B2 have fewer virulence factors than their susceptible counterparts. J Clin Microbiol. 2005;43(6):2962-4.

17. Arisoy M, Rad AY, Akin A, Akar N. Relationship between susceptibility to antimicrobials and virulence factors in paediatric Escherichia coli isolates. Int J Antimicrob Agents. 2008;31 Suppl 1:S4-8.

18. Zhao L, Chen X, Zhu X, Yang W, Dong L, Xu X, et al. Prevalence of virulence factors and antimicrobial resistance of uropathogenic Escherichia coli in Jiangsu province (China). Urology. 2009;74(3):702-7. 